\title{
A nanoflare model for active region radiance: application of artificial neural networks
}

\author{
M. Bazarghan ${ }^{1,2}$, H. Safari ${ }^{2,3,4}$, D. E. Innes ${ }^{3}$, E. Karami ${ }^{5}$, and S. K. Solanki ${ }^{3}$ \\ 1 IUCAA, Post Bag 4, Ganeshkhind, Pune 411 007, India \\ 2 Institute for Advanced Studies in Basic Sciences, Zanjan, Iran \\ 3 Max-Planck Institut für Sonnensystemforschung, 37191 Katlenburg-Lindau, Germany \\ e-mail: innes@mps .mpg . de \\ 4 Department of Physics, Zanjan University, Zanjan, Iran \\ 5 Department of Electronics Science, University of Pune, Pune 411007, India
}

Received 4 September 2008 / Accepted 22 October 2008

\begin{abstract}
Context. Nanoflares are small impulsive bursts of energy that blend with and possibly make up much of the solar background emission. Determining their frequency and energy input is central to understanding the heating of the solar corona. One method is to extrapolate the energy frequency distribution of larger individually observed flares to lower energies. Only if the power law exponent is greater than 2 is it considered possible that nanoflares contribute significantly to the energy input.

Aims. Time sequences of ultraviolet line radiances observed in the corona of an active region are modelled with the aim of determining the power law exponent of the nanoflare energy distribution.

Methods. A simple nanoflare model based on three key parameters (the flare rate, the flare duration, and the power law exponent of the flare energy frequency distribution) is used to simulate emission line radiances from the ions Fe XIX, Ca xIII, and Si III, observed by SUMER in the corona of an active region as it rotates around the east limb of the Sun. Light curve pattern recognition by an Artificial Neural Network (ANN) scheme is used to determine the values.

Results. The power law exponents, $\alpha \approx 2.8,2.8$, and 2.6 are obtained for Fe XIX, Ca XIII, and Si III respectively.

Conclusions. The light curve simulations imply a power law exponent greater than the critical value of 2 for all ion species. This implies that if the energy of flare-like events is extrapolated to low energies, nanoflares could provide a significant contribution to the heating of active region coronae.
\end{abstract}

Key words. Sun: activity - Sun: flares - Sun: UV radiation

\section{1. introduction}

Heating the corona by the dissipation of current sheets was first suggested by Gold (1964) and later developed to form the basis of the nanoflare heating model by Levine (1974) and Parker $(1983,1988)$. The idea is that current sheets arise spontaneously in coronal magnetic fields that are braided and twisted by random photospheric footpoint motions. These current sheets dissipate in many small-scale reconnection events, heating and accelerating plasma in the coronal loops. In the corona, they would give rise to multiple unresolvable loop strands with specific observable signatures (Zirker \& Cleveland 1994; Warren et al. 2002; Cargill \& Klimchuk 2004; Patsourakos \& Klimchuk 2005). Recently Aschwanden (2008) found evidence against such multi-temperature strands in TRACE coronal images. He concludes that nanoflare heating is only possible if it occurs in the chromosphere/transition region where heating across magnetic field lines can produce the isothermal loops seen in the corona. Irrespective of where the nanoflare energy input sites are, a key question is whether the energy of nanoflares is sufficient to heat the corona or not. Most of the individual nanoflares would be too small to detect and the majority would be small fluctuations on the overall background. That background could be produced by the blending of many small events.
The approach taken to estimate their contribution has been to extrapolate the energy frequency distribution of detectable flarelike events. The energy frequency distribution of larger flares tends to follow a power law distribution

$\frac{\mathrm{d} N}{\mathrm{~d} E} \sim E^{-\alpha}$,

where $\mathrm{d} N$ is the number of flares per energy interval $\mathrm{d} E$. The energy of small flares dominates if $\alpha>2$ (Hudson 1991). This is therefore a critical parameter for the nanoflare heating model. The standard method to determine $\alpha$ is to evaluate the energy of many flares in a series of observations and then plot their frequency in bins of energy $\mathrm{d} E$. The majority of analyses based on this type of event counting deduce $\alpha \approx 1.7$ (Lin et al. 1984; Shimizu 1995; Aschwanden \& Parnell 2002), a value smaller than the critical 2. These results may, however, be misleading. For example, Parnell (2004) demonstrated that one can obtain $\alpha$ ranging from 1.5 to 2.6 for the same data set using different but still reasonable sets of assumptions for the analyses.

Here we take an alternative approach and model ultraviolet (UV) radiances observed by the Solar Ultraviolet Measurements of Emitted Radiation (SUMER; Wilhelm et al. 1995, 1997) in an active region corona, assuming that the radiance fluctuations and the nearly constant "background" emission are caused by small-scale stochastic flaring (Pauluhn \& Solanki 2004, 2007). 


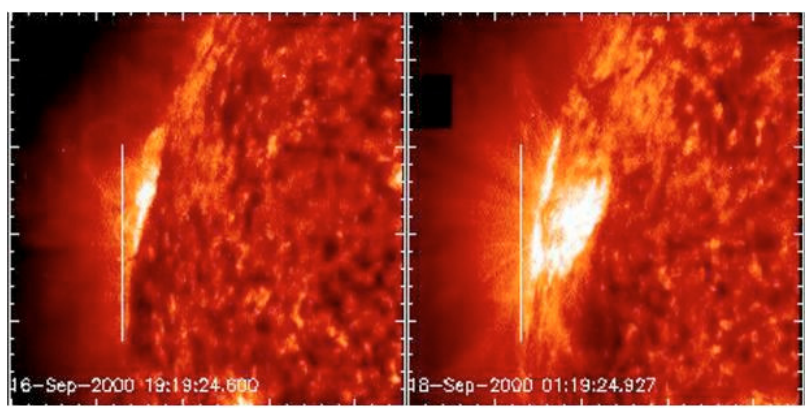

Fig. 1. EIT $195 \AA$ images of the observed active region at two times, showing the position of the SUMER slit, indicated by the vertical line.

The model has been applied successfully to UV radiance fluctuations in the quiet Sun (Pauluhn \& Solanki 2007). The method compares light curves generated assuming random flaring with a power law frequency distribution to the light curves of an observed emission line. It has the advantage that it takes into account without bias weak, blended micro- and nanoflares that produce a nearly continuous background.

Here we apply this technique to off-limb time series recorded by SUMER. The three lines modelled, Fe XIX $\lambda 1118.07$ (6.3 MK), Ca XIII $\lambda 1133.76$ (2.2 MK) and Si III $\lambda 1113.23$ (0.06 MK), cover two decades of formation temperature from the lower transition region to the hotter gas in the corona.

The analysis described here uses Artificial Neural Networks (ANNs) to find the optimum match to the three parameters of the model. The main advantage of this method over previous analyses based on the radiance distribution function (Pauluhn \& Solanki 2007; Safari et al. 2007) is that we are able to obtain quantitative values for all parameters, including $\alpha$. Another advantage of the ANN method is that it concentrates on the number and shape of the emission peaks along the light curves with little weight on the low radiance pixels, which was a problem with the Safari et al. (2007) analysis.

\section{SUMER data and analysis}

The observed active region (AR 1967) is shown in Fig. 1. This is the region and data set discussed in Wang et al. (2006). The SUMER $300^{\prime \prime} \times 4^{\prime \prime}$ slit was placed, as shown, at a fixed position above the limb. Observations with a cadence of $90 \mathrm{~s}$ in six spectral lines, Fe XIX $\lambda 1118.07$ (6.3 MK), Ca XV $\lambda 1098.48$ and $\lambda 555.38$ (3.5 MK), Ca XIII $\lambda 1133.76$ (2.2 MK), Ne vi $\lambda 558.62$ (0.3 MK) and Si III $\lambda 1113.23$ (0.06 MK) were transmitted, for periods of $12.6 \mathrm{~h}$ followed by a full spectrum scan $(800-1600 \AA)$ of $3.4 \mathrm{~h}$. A typical time sequence in any one line consists of 500 exposures. The three strongest lines, Fe XIX, Ca XIII, and Si III, are analysed here. Images of their radiance along the slit are shown in Fig. 2 for a typical $12.6 \mathrm{~h}$ period. Distinct events can be seen in Fe XIx, but only the very strongest make an impression on the bright active region Ca XIII emission when they cool (Innes \& Wang 2004). Si III is seen close to the limb and appears to be generated by small surge-like ejections. Our results are based on three such time series, taken over the days 16-18 September 2000.

The emission along each row was very noisy at several positions. To improve the signal-to-noise but at the same time not to lose individual structures, the light curves were obtained by first averaging SUMER data over five spatial pixels $\left(5^{\prime \prime}\right)$ along the slit. Only the light curves with all 500 data points above a chosen threshold were selected for analysis. We did not want to

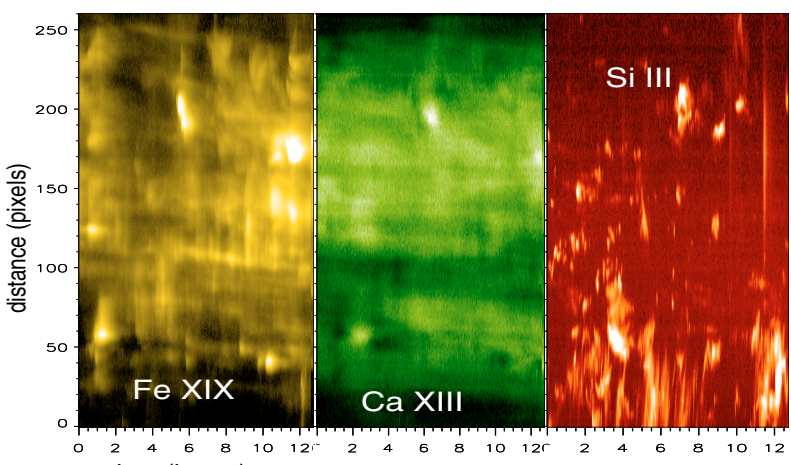

time (hours)

Fig. 2. Time series of line radiance along the SUMER spectrometer slit for the period 16 Sep. 19:00 UT to 17 Sep. 7:36 UT. The distance in pixels along the SUMER slit is shown on the vertical axis.

base the threshold on an absolute intensity because this would have biased the input data against low background. So for the Fe XIX and Ca XIII, the threshold was set such that the ratio of the Ne vi to Fe XIX intensity was less than 0.5 . This ensures that only light curves from the central part of the active region were taken. Most of the Si III emission was concentrated near the limb to the south (Fig. 2). The Si III selection was based on the local scatter in the second moment of the line, the line width. If the standard deviation of the line width was greater than 1.0 over a local $5 \times 5$ space-time block, then the central data point and associated light curve were excluded from the analysis. This resulted in 35 test light curves for both Fe XIX and Ca XIII and 11 test curves for Si III. Before being fed to the neural network, all light curves were normalized to their maximum.

\section{Model}

\subsection{Applicability}

The emission in the active region corona is assumed to be caused by many random flares with flare radiances following a power law frequency distribution. Flares with a power law frequency distribution, $\alpha$, in radiance are assumed to erupt with a frequency, $p_{f}$, and have a flare duration $\tau=\tau_{\mathrm{r}}+\tau_{\mathrm{d}}$, where $\tau_{\mathrm{r}}$ is the rise time and $\tau_{\mathrm{d}}$ the decay time. We assume $\tau_{\mathrm{r}} / \tau_{\mathrm{d}}=0.5$. The other free parameter in the model is the ratio of the maximum to minimum flare energy which is set to $E_{\max } / E_{\min }=50$.

For a large number of independent random flares, the distribution of normalized radiances, $J=I / \bar{I}$ where $I$ is the radiance, is lognormal with shape parameter $\sigma$ (Pauluhn \& Solanki 2007):

$$
f(J)=\frac{1}{\sigma J \sqrt{2 \pi}} \exp \left(-\frac{(\log J)^{2}}{2 \sigma^{2}}\right) .
$$

$\sigma$ is inversely proportional to $\sqrt{\tau p_{f}}$ (Pauluhn \& Solanki 2007), with a slight $\alpha$ dependence (Safari et al. 2007). A small shape parameter $(\sigma<0.3)$ indicates a symmetric distribution due to high background emission caused by either a long duration time, $\tau$, or a high flare frequency, $p_{f}$. The radiance distributions of the three lines of Fe xIX, Ca XIII and Si III and their lognormal fits are shown in Fig. 3. This gives us confidence that the stochastic flare model is applicable. It is interesting to note that both the Fe XIX and the Si III lines have the same shape parameter.

Light curves for the stochastic flare model are shown for $\alpha=$ 1.6 and $\alpha=2.4$, and two combinations of $\tau p_{f}=2$ in Fig. 4. The light curves are visibly different, although they all have shape parameter $\sigma \approx 0.6$. The effect of $\alpha$ on the light curve is seen in 

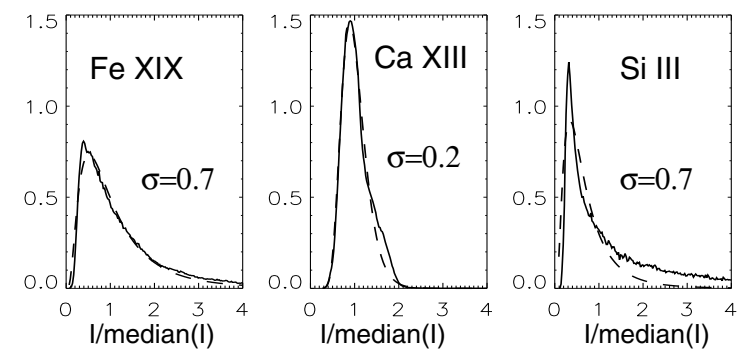

Fig. 3. Distribution functions of SUMER data in the active region corona (solid lines) and best fit lognormal functions (dashed lines). The radiances are normalized to their median and their distributions to the number of data points.
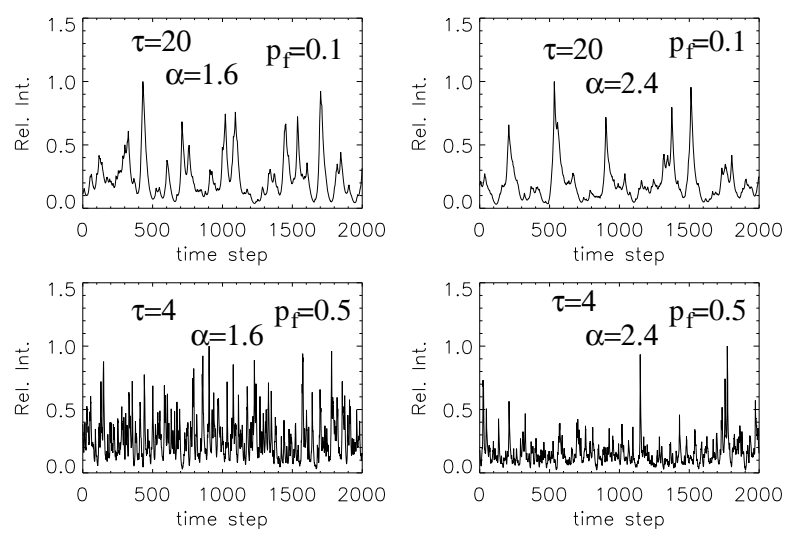

Fig. 4. Light curves for flare models run with different $\tau, p_{f}$ and $\alpha$ parameters. All light curves have $\tau p_{f}=2$.

the ratio of strong to weak flares. The left-hand light curves have more large flares because they have a smaller exponent. Picking up these pattern changes is the strength of the ANN method.

\subsection{Neural networks and parameter estimation}

We applied the ANN method to probe the unknown parameters (power law exponent, $\alpha$, duration time, $\tau$, and flare rate, $p_{f}$ ) of the three lines. ANNs have become a popular tool in almost every field of science. In recent years, ANNs have been widely used in astronomy for applications such as star/galaxy discrimination, (Andreon et al. 2000; Cortiglioni et al. 2001), morphological classification of galaxies, (Storrie-Lombardi et al. 1992; Ball et al. 2004), and spectral classification of stars (von Hippel et al. 1994; Bazarghan \& Gupta 2008; Bazarghan 2008).

We employ probabilistic neural networks (PNNs Specht 1988, 1990). The PNN learns to approximate the probability density function of the training samples. It uses a supervised training set to develop distribution functions within a pattern layer. These functions in the recall mode are used to estimate the likelihood of an input feature vector being part of a learned category or class.

An example of a PNN is shown in Fig. 5. This network has four layers. The network contains an input layer which has as many elements as there are separable parameters needed to describe the objects to be classified. It has a pattern layer, which organizes the training set such that each input vector is represented by an individual processing element. The third layer is the summation layer, which has as many processing elements as there are classes to be recognized. Each element in this layer combines via processing elements with the pattern layer which

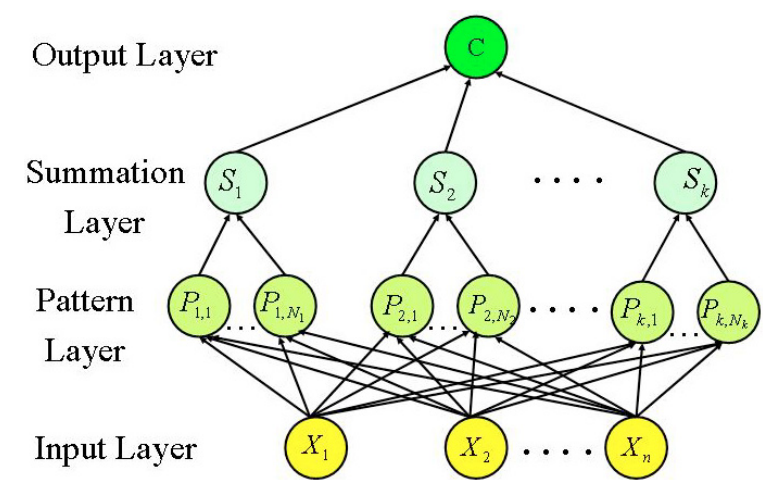

Fig. 5. Schematic of a typical probabilistic neural network.

relates to the same class and prepares that category for output. Finally, there is the output layer that corresponds to the summation unit with the maximum output.

For the identification of SUMER light curves, the input vector, $X=\left(x_{1}, x_{2}, \ldots, x_{n}\right)$, is the light curve with 500 data points $(n=500)$. The network is first trained to classify light curves corresponding to all the possible combinations of $\alpha, \tau$, and $p_{f}$. For this we synthetically generate light curves with the nanoflare code described in Pauluhn \& Solanki (2007). We generate one light curve for each combination of the parameters:

- the power law exponent spanning $1.5 \leq \alpha \leq 3.2$ in steps of 0.1 ;

- the duration time spanning $1.5 \leq \tau \leq 52$ in steps of 1 ;

- the flare rate spanning $0.1 \leq p_{f} \leq 0.9$ in steps of 0.1 with additional values at 0.05 and 0.95 .

This gives a set of 6930 pattern groups $(k=6930)$, one group for each combination of $\alpha, \tau$, and $p_{f}$. Each pattern group, $k$, is characterized by $N_{k}$ Gaussian functions (Specht 1988, 1990).

When a SUMER light curve of an unknown classification is fed to the network, the summation layer of the network computes the probability functions $S_{k}$ of each class. Finally at the output layer we have $C$, the value with the highest probability.

\section{Results and conclusions}

\subsection{Results}

In the present work, PNN is used as a tool to extract the three flare model parameters required to reproduce the SUMER light curves. All $35 \mathrm{Fe}$ XIX and CaxIII, and 11 Si III SUMER light curves from the three days of observations were fed individually into the neural network and the parameters were obtained for each light curve separately. The final PNN outputs are shown in Table 1 . The bold numbers are the statistically maximum occurrence for each parameter. For example for Fe XIX, $\alpha=2.8$ is found in more than $70 \%$ of the light curves. The minimum and maximum values, given on the left and right, indicate the scatter in the light curve parameters.

In each line there is $20 \%$ scatter in $\alpha$, and $50 \%$ scatter in $\tau$. The range of $p_{f}$ values for Fe XIX and Si III is much broader, suggesting that events producing emission in these temperature ranges do not have the same rate everywhere but are seen in irregular bursts. We also note that the value of $\tau p_{f}$ is roughly the same for both Fe XIX and Si III, as suggested by their shape parameter (Fig. 3). The CaXIII light curves are all matched with a high value of $p_{f}$, consistent with the idea that the $1 \mathrm{MK}$ active region corona requires almost continuous flaring. The four 
Table 1. The SUMER spectral lines and the parameter values given by PNN.

\begin{tabular}{|c|c|c|c|}
\hline SUMER spectra & \multicolumn{3}{|c|}{ PNN outputs ${ }^{1}$} \\
\hline lines & $\alpha$ & $\tau$ & $p_{f}$ \\
\hline Fe XIX & 2.52 .83 .1 & 91420 & $\begin{array}{llll}0.1 & \mathbf{0 . 2} & 0.7\end{array}$ \\
\hline Ca XIII & 2.62 .83 .0 & 414545 & $0.8 \mathbf{0 . 9} 0.9$ \\
\hline Si III & 2.42 .62 .8 & 5912 & $\begin{array}{llll}0.2 & \mathbf{0 . 3} & 0.9\end{array}$ \\
\hline
\end{tabular}

1 The most frequent values are given in bold, and the minimum and maximum on the left and right. $\tau$ is per exposure time (90 s) and $p_{f}$ is per exposure time per $5^{\prime \prime} \times 4^{\prime \prime}$ spatial element.

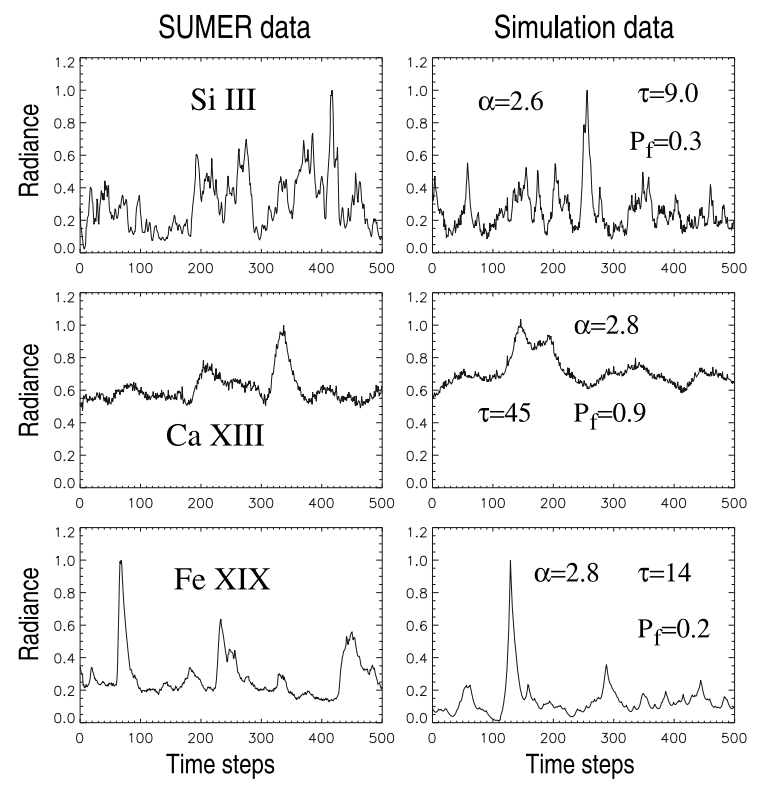

Fig. 6. Samples of the radiance time series: left panel: SUMER data, and right panel: simulation data obtained with the parameters given in Table 1.

times higher rate for Ca XIII than Fe XIX suggests that most of the $\mathrm{Ca}$ XIII emission is produced by heating events below the Fe XIX formation temperature (6.6 MK).

Example light curves obtained using these parameters are compared with the observed ones in Fig. 6. Both the Si III and Ca XIII simulations look remarkably similar to their observed light curves. The background radiance of the Fe XIx light curve is about a factor of 2 too low. The Fe xIx light curves had a $p_{f}$ ranging from 0.7 to 0.1 , so we suspect that in this case the $p_{f}$ value is slightly too low. Also for Fe XIX, the ratio $\tau_{\mathrm{r}} / \tau_{\mathrm{d}}$ deduced from the data is smaller than the fixed value 0.5 used here. This may influence the accuracy of the method.

The sensitivity of the PNN output depends on the training set. During the training session, the network must see all possible patterns that it is supposed to classify in the testing session. With 500 simulated light curves in the training set, PNN was not able to converge for several of the SUMER light curves. When we increased the number of simulated light curves to 6930, we were able to obtain unique parameters for all observed light curves.

\subsection{Conclusions}

The concept that the solar corona may be heated by numerous, randomly distributed, small flare-like events called nanoflares is considered by comparing simulated and observed emission line light curves. The difference between this and previous methods is the fully automated modelling of the light curve structure. There is no human decision required for background/event cutoff levels or best fit parameters.

The result is power law flare energy frequency exponents greater than 2.5 for all three emission lines considered, Si III, CaXIII and FexIX. This is consistent with the corona being heated mainly by nanoflares, and demonstrates the importance of nanoflare "background" emission in determining the power law exponents. The parameter with highest uncertainty or largest scatter is the flare rate, especially for the lines formed at transition region and hot flare temperatures. Coronal plasma at these temperatures is produced sporadically and is associated with more specific coronal and chromospheric loop structures than the general active region corona, so the scatter is to be expected.

The next step will be to determine the actual flare energies producing the nanoflare emission. This is a much more complicated exercise because the modelled light curves are observed in the corona which may be heated by events occurring lower in the atmosphere (Aschwanden 2008), so that it requires a model for the energy transfer to the observation position.

Acknowledgements. H. Safari acknowledges the warm hospitality and financial support during his research visit to the solar group, MPS.

\section{References}

Andreon, S., Gargiulo, G., Longo, G., Tagliaferri, R., \& Capuano, N. 2000, MNRAS, 319, 700

Aschwanden, M. J. 2008, ApJ, 672, L135

Aschwanden, M. J., \& Parnell, C. E. 2002, ApJ, 572, 1048

Ball, N. M., Loveday, J., Fukugita, M., et al. 2004, MNRAS, 348, 1038 Bazarghan, M. 2008, Bulletin of the Astronomical Society of India, 36, 1 Bazarghan, M., \& Gupta, R. 2008, Ap\&SS

Cargill, P. J., \& Klimchuk, J. A. 2004, ApJ, 605, 911

Cortiglioni, F., Mähönen, P., Hakala, P., \& Frantti, T. 2001, ApJ, 556, 937

Gold, T. 1964, in The Physics of Solar Flares, 389

Hudson, H. S. 1991, Sol. Phys., 133, 357

Innes, D. E., \& Wang, T. J. 2004, in ESA SP-575: SOHO 15 Coronal Heating, 553

Levine, R. H. 1974, ApJ, 190, 457

Lin, R. P., Schwartz, R. A., Kane, S. R., Pelling, R. M., \& Hurley, K. C. 1984, ApJ, 283, 421

Parker, E. N. 1983, ApJ, 264, 642

Parker, E. N. 1988, ApJ, 330, 474

Parnell, C. E. 2004, in ESA SP-575: SOHO 15 Coronal Heating, 227

Parzen, E. 1962, Ann. Math. Stat., 33, 1065

Patsourakos, S., \& Klimchuk, J. A. 2005, ApJ, 628, 1023

Pauluhn, A., \& Solanki, S. K. 2004, in ESA SP-575: SOHO 15 Coronal Heating, ed. R. W. Walsh, J. Ireland, D. Danesy, \& B. Fleck, 501

Pauluhn, A., \& Solanki, S. K. 2007, A\&A, 462, 311

Safari, H., Innes, D. E., Solanki, S. K., \& Pauluhn, A. 2007, in Modern solar facilities - advanced solar science, 2006, Universitätsverlag Göttingen, ed. F. Kneer, K. G. Puschmann, \& A. D. Wittmann, 359

Shimizu, T. 1995, PASJ, 47, 251

Specht, D. F. 1988, in Proc. IEEE International Conference on Neural Networks, San Diego, CA, 1, 525

Specht, D. F. 1990, Neural Networks, 3, 109

Storrie-Lombardi, M. C., Lahav, O., Sodre, Jr., L., \& Storrie-Lombardi, L. J. 1992, MNRAS, 259, 8

von Hippel, T., Storrie-Lombardi, L. J., Storrie-Lombardi, M. C., \& Irwin, M. J. 1994, MNRAS, 269, 97

Wang, T. J., Innes, D. E., \& Solanki, S. K. 2006, A\&A, 455, 1105

Warren, H. P., Winebarger, A. R., \& Hamilton, P. S. 2002, ApJ, 579, L41

Wilhelm, K., Curdt, W., Marsch, E., et al. 1995, Sol. Phys., 162, 189

Wilhelm, K., Lemaire, P., Curdt, W., et al. 1997, Sol. Phys., 170, 75

Zirker, J. B., \& Cleveland, F. M. 1994, Sol. Phys., 153, 245 